Pacific Journal of Mathematics

SOME GENERALIZATIONS OF CONTRACTION THEOREMS
FOR FOURIER SERIES 


\section{SOME GENERALIZATIONS OF CONTRACTION THEOREMS FOR FOURIER SERIES}

\section{MaSAKITI KINUKAWA}

This paper deals with some contraction theorems for Fourier series and certain properties of the Fourier coefficients of functions in the Lipschitz spaces.

1. We shall assume that functions $f$ and $g$ are integrable in $(-\pi, \pi)$ and periodic with period $2 \pi$. Denote their Fourier series by

$$
f \sim \sum_{n=-\infty}^{\infty} c_{n} e^{i n x}, \text { and } g \sim \sum_{n=-\infty}^{\infty} d_{n} e^{i n x} .
$$

According to B. S. Yadav [10], we say a function $g$ is a shrivel contraction of order $j$ of a function $f$ if it holds that

$$
\left|L^{(j)}(t, x, g)\right| \leq K\left|L^{(j)}(t, x, f)\right|,
$$

where

$$
L^{(j)}(t, x, f)=\frac{1}{t} \int_{0}^{t} \Delta_{u}^{J} f(x) d u
$$

and

$$
\Delta_{u}^{j} f(x)=\sum_{m=0}^{j}(-1)^{j+m}\left(\begin{array}{c}
j \\
m
\end{array}\right) f(x+(j-2 m) u),
$$

which is the symmetric difference of order $j$ of $f(x)$ with respect to $u$.

Yadav proved the following:

THEOREM Y. If $g$ is a shrivel contraction of order $j$ of $f$ and if

$$
{ }_{2} B_{p}\left(c_{n}\right)=\left[\sum_{\substack{n=-\infty \\ n \neq 0}}^{\infty}|n|^{-p / 2}\left(\sum_{|k| \geq n+1}\left|c_{k}\right|^{2}\right)^{p / 2}\right]^{1 / p}<\infty,
$$

then

$$
\left\|d_{n}\right\|_{p}^{p}=\sum_{n=-\infty}^{\infty}\left|d_{n}\right|^{p}<\infty, \text { where } 0<p \leq 2
$$


Remark that (2) implies $\left\|c_{n}\right\|_{p}^{p}=\sum_{n=-\infty}^{\infty}\left|c_{n}\right|^{p}<\infty$. (See, for example, Yadav's paper.) Therefore, Yadav's theorem means that the shrivel contraction (1) operates on the class of functions whose Fourier series are in the class $l_{p}$ with ${ }_{2} B_{p}\left(c_{n}\right)<\infty$.

We shall give more precise results than Yadav's theorem and make the situation clear.

2. Throughout the paper, we use the following notations:

$$
\begin{aligned}
{ }_{a} B_{p, \alpha}\left(c_{n}\right) & =\left\{\sum_{k=1}^{\infty} k^{p \alpha-1}\left[\sum_{|n| \geq k}\left|c_{n}\right|^{a}\right]^{p / a}\right\}^{1 / p}, \\
{ }_{a} C_{p, j, \alpha}\left(c_{n}\right) & =\left\{\sum_{k=1}^{\infty} k^{p \alpha-1-p j}\left[\sum_{0<|n| \leq k}\left|c_{n}\right|^{a}|n|^{a j}\right]^{p / a}\right\}^{1 / p}, \\
{ }_{a} A_{p, J, \alpha}(f) & =\left\{\int_{0}^{1}\left[\left.\int_{-\pi}^{\pi}\left|\frac{\Delta_{t}^{J} f(x)}{|t|^{\alpha}}\right|^{a} d x\right|^{p / a} \frac{d t}{t}\right\}^{1 / p},\right. \\
{ }_{a} \mathbf{A}_{p, j, \alpha}(f) & =\left\{\int_{0}^{1}\left[\int_{-\pi}^{\pi}\left|\frac{L^{(j)}(t, x, f)}{|t|^{\alpha}}\right|^{a} d x\right]^{p / a} \frac{d t}{t}\right\}^{1 / p}, \\
\mathbf{Y}_{a, j}\left(t, c_{n}\right) & =\left[\sum_{n=-\infty}^{\infty}\left|c_{n}\right|^{a}\left|\frac{1}{t} \int_{0}^{t}(\sin n u)^{J} d u\right|^{a}\right]^{1 / a}, \\
{ }_{a} \tilde{\mathbf{A}}_{p, j, \alpha}\left(c_{n}\right) & =\left\{\int_{0}^{1}\left[\frac{\mathbf{Y}_{a, j}\left(t, c_{n}\right)}{|t|^{\alpha}}\right]^{p} \frac{d t}{t}\right\}^{1 / p} \cdot
\end{aligned}
$$

Lemma 1. Let $0<p<a<\infty, 0<\alpha<j<\infty$. The finiteness of ${ }_{a} \tilde{\mathbf{A}}_{p, J, \alpha}\left(c_{n}\right),{ }_{a} B_{p, \alpha}\left(c_{n}\right)$, and ${ }_{a} C_{p, j, \alpha}\left(c_{n}\right)$ are mutually equivalent; that is, we have

$$
\begin{aligned}
{ }_{a} \tilde{\mathbf{A}}_{p, j, \alpha}\left(c_{n}\right) & \leq K\left\{{ }_{a} B_{p, \alpha}\left(c_{n}\right)+{ }_{a} C_{p, j, \alpha}\left(c_{n}\right)\right\} . \\
{ }_{a} C_{p, j, \alpha}\left(c_{n}\right) & \leq K\left\{{ }_{a} B_{p, \alpha}\left(c_{n}\right)\right\} . \\
{ }_{a} B_{p, \alpha}\left(c_{n}\right) & \leq K\left\{{ }_{a} C_{p, j, \alpha}\left(c_{n}\right)\right\} . \\
{ }_{a} C_{p, j, \alpha}\left(c_{n}\right) & \leq K\left\{{ }_{a} \tilde{\mathbf{A}}_{p, j, \alpha}\left(c_{n}\right)\right\} .
\end{aligned}
$$

( $K=$ Positive constant numbers which may be different from one occurrence to another.) 
Proof. Let us prove the first inequality. By the definition, we have

$$
\begin{aligned}
& \left\{{ }_{a} \tilde{\mathbf{A}}_{p, j, \alpha}\left(c_{n}\right)\right\}^{p} \\
& \quad=\int_{0}^{1} t^{-p \alpha-1}\left[\sum_{n=-\infty}^{\infty}\left|c_{n}\right|^{a}\left|\frac{1}{t} \int_{0}^{t}(\sin n u)^{j} d u\right|^{a}\right]^{p / a} d t \\
& \quad \leq K \int_{0}^{1} t^{-p \alpha-1}\left\{\left[\sum_{|n| \leq 1 / t}\left|c_{n}\right|^{a}|n t|^{a j}\right]^{p / a}+\left[\sum_{|n|>1 / t}\left|c_{n}\right|^{a}\right]^{p / a}\right\} d t \\
& \quad=K\left(I_{1}+I_{2}\right), \text { say. }
\end{aligned}
$$

Then we have

$$
\begin{aligned}
I_{1} & =K \sum_{k=1}^{\infty} \int_{1 /(k+1)}^{1 / k} t^{-p \alpha-1+p j}\left[\sum_{|n| \leq 1 / t}\left|c_{n}\right|^{a}|n|^{a_{J}}\right]^{p / a} d t \\
& \leq K \sum_{k=1}^{\infty} k^{p \alpha-1-p J}\left[\sum_{|n| \leq k}\left|c_{n}\right|^{a}|n|^{a_{j}}\right]^{p / a}
\end{aligned}
$$

which is dominated by $K\left\{{ }_{a} C_{p, j, \alpha}\left(c_{n}\right)\right\}^{p}$. In a similar way, we can estimate the second part by $K\left\{{ }_{a} B_{p, \alpha}\left(c_{n}\right)\right\}^{p}$ (cf. G. Sunouchi [9]).

Let us prove (4):

$$
\begin{aligned}
\left\{{ }_{a} C_{p, j, \alpha}\left(c_{n}\right)\right\}^{p} & \leq K \sum_{k=0}^{\infty}\left(2^{k}\right)^{p \alpha-p j} \sum_{m=0}^{k}\left[\sum_{2^{m} \leq|n| \leq 2^{m+1}}\left|c_{n}\right|^{a}|n|^{a j}\right]^{p / a} \\
& \leq K \sum_{m=0}^{\infty}\left(2^{m}\right)^{p j}\left(\sum_{2^{m} \leq|n| \leq 2^{m+1}}\left|c_{n}\right|^{a}\right)^{p / a} \sum_{k=m}^{\infty}\left(2^{k}\right)^{p \alpha-p J} \\
& \leq K \sum_{m=0}^{\infty}\left(2^{m}\right)^{p \alpha}\left[\sum_{|n| \geq 2^{m}}\left|c_{n}\right|^{a}\right]^{p / a}
\end{aligned}
$$

which is convergent if ${ }_{a} B_{p, \alpha}\left(c_{n}\right)$ is finite.

Let us prove (5).

$$
\left\{{ }_{a} B_{p, \alpha}\left(c_{n}\right)\right\}^{p} \leq K \sum_{k=0}^{\infty}\left(2^{k}\right)^{p \alpha} \sum_{m=k}^{\infty}\left(2^{m}\right)^{-p j}\left[\sum_{2^{m} \leq|n| \leq 2^{m+1}}\left|c_{n}\right|^{a}|n|^{a j}\right]^{p / a} .
$$

Interchanging the order of summations, we have

$$
\leq K \sum_{m=0}^{\infty}\left(2^{m}\right)^{-p_{J}+p \alpha}\left[\sum_{|n| \leq 2^{m+1}}\left|c_{n}\right|^{a}|n|^{a_{J}}\right]^{p / a}
$$

which is finite if ${ }_{a} C_{p, J, \alpha}\left(c_{n}\right)$ is finite. 
Let us prove (6).

$\left\{{ }_{a} \tilde{\mathbf{A}}_{p, J, \alpha}\left(c_{n}\right)\right\}^{p} \geq \sum_{k=1}^{\infty} \int_{1 / 2(k+1)}^{1 / 2 k} t^{-p \alpha-1}\left[\sum_{|n| \leq k}\left|c_{n}\right|^{a}\left|\frac{1}{t} \int_{0}^{t}(\sin n u)^{j} d u\right|^{a}\right]^{p / a} d t$.

Since $0 \leq|n u| \leq k t \leq k / 2 k \leq \pi / 2$, we have

$$
\left|\frac{1}{t} \int_{0}^{t}(\sin n u)^{j} d u\right| \geq K \mid n t \psi .
$$

Therefore, we have

$$
\begin{aligned}
\left\{{ }_{a} \tilde{\mathbf{A}}_{p, j, \alpha}\left(c_{n}\right)\right\}^{p} & \geq K \sum_{k=1}^{\infty} \int_{1 / 2(k+1)}^{1 / 2 k} t^{-p \alpha-1+p_{J}} d t\left[\sum_{|n| \leq k}\left|c_{n}\right|^{a}|n|^{a j}\right]^{p / a} \\
& \geq K \sum_{k=1}^{\infty} k^{p \alpha-p j-1}\left[\sum_{|n| \leq k}\left|c_{n}\right|^{a}|n|^{a j}\right]^{p / a} \\
& =K\left\{{ }_{a} C_{p, J, \alpha}\left(c_{n}\right)\right\}^{p} .
\end{aligned}
$$

THEOREM 1. The following finiteness of norms are equivalent:

$$
{ }_{2} \mathbf{A}_{p, j, \alpha}(f)<\infty, \quad{ }_{2} B_{p, \alpha}\left(c_{n}\right)<\infty, \quad{ }_{2} C_{p, J, \alpha}\left(c_{n}\right)<\infty,
$$

where $0<p<2,0<\alpha<j$.

Proof. The Fourier series of $\Delta_{u}^{J} f(x)$ is

$$
\Delta_{u}^{j} f(x) \sim K \sum_{n=-\infty}^{\infty} c_{n} e^{i n x}(\sin n u)^{J}
$$

and so

$$
L^{(J)}(t, x, f) \sim K \sum_{n=-\infty}^{\infty} c_{n} e^{i n x} \frac{1}{t} \int_{0}^{t}(\sin n u)^{j} d u
$$

By the Parseval theorem, we have

$$
\left\{\int_{-\pi}^{\pi}\left|L^{(j)}(t, x, f)\right|^{2} d x\right\}^{1 / 2}=K \mathbf{Y}_{2, j}\left(t, c_{n}\right)
$$

that is,

$$
{ }_{2} \mathbf{A}_{p, j, \alpha}(f)=K_{2} \tilde{\mathbf{A}}_{p, j, \alpha}\left(c_{n}\right) .
$$

Now, Theorem 1 is followed from Lemma 1. 
Yadav's result is a corollary of Theorem 1. In fact, we have a more general result:

THEOREM 2. Suppose the integers $l$ and $j$ are greater than $1 / p-1 / 2$. If a general shrivel contraction

$$
\left|L^{(l)}(t, x, g)\right| \leq K\left|L^{(j)}(t, x, f)\right|
$$

holds for all $x$ and for all $t>0$, and if ${ }_{2} B_{p, \alpha}\left(c_{n}\right)<\infty$ for $\alpha=1 / p-1 / 2$, then

$$
\sum_{n=-\infty}^{\infty}\left|d_{n}\right|^{p}<\infty
$$

Proof. Note that Yadav's condition (2) is equivalent to ${ }_{2} B_{p, \alpha}\left(c_{n}\right)<\infty$ for $\alpha=1 / p-1 / 2$. By Theorem 1, we have ${ }_{2} \mathbf{A}_{p, j, \alpha}(f)<\infty$. However, the condition (7) implies

$$
{ }_{2} \mathbf{A}_{p, l, \alpha}(g) \leq K_{2} \mathbf{A}_{p, j, \alpha}(f)<\infty .
$$

According to Theorem $1,{ }_{2} B_{p, \alpha}\left(d_{n}\right)<\infty$, by which we have $\sum_{n=-\infty}^{\infty}\left|d_{n}\right|^{p}$ $<\infty$.

We should add another remarks: In the previous paper [4], we mentioned that ${ }_{2} A_{p, J, \alpha}(f)<\infty$ is equivalent to ${ }_{2} B_{p, \alpha}\left(c_{n}\right)<\infty$. Therefore, ${ }_{2} \mathbf{A}_{p, j, \alpha}(f)<\infty$ is equivalent to ${ }_{2} A_{p, j, \alpha}(f)<\infty$. Also, we proved that ${ }_{2} A_{p, j, \alpha}(f)<\infty$ is equivalent to ${ }_{2}\left\|c_{n}\right\|_{p}<\infty$, which is the Beurling's norm given in the next section (cf. [1]). Therefore, we have that ${ }_{2} \mathbf{A}_{p, j, \alpha}(f)$ $<\infty$ is equivalent to ${ }_{2}\left\|c_{n}\right\|_{p}<\infty$.

3. In this section, we shall discuss some generalizations of the previous results [4]. Before going into details, we should remark the following: We have denoted $\Delta_{u}^{j} f(x)$ the symmetric difference of $f(x)$. This is somewhat essential in the case of shrivel contraction arguments. That is, if we replace the symmetric difference by the ordinary one, then the part of integration in the definition of $\mathbf{Y}_{a, j}\left(t, c_{n}\right)$ must be read as $\int_{0}^{t}\left(e^{i n u}-1\right)^{j} d u$, and we meet some difficulties in the arguments for the inequality (6).

However, in what follows, the symmetrization has no essential role. Therefore, in this section, we shall adopt the ordinary difference $\Delta_{t}^{j} f(x)$, that is,

$$
\Delta_{t}^{j} f(x)=\sum_{m=0}^{j}(-1)^{j+m}\left(\begin{array}{c}
j \\
m
\end{array}\right) f(x+m t) .
$$


We also use the following notations:

$I(t)=$ a positive function which is increasing on $(0,1)$.

$$
\begin{gathered}
{ }_{a} A_{p, J, I}(f)=\left\{\int_{0}^{1}\left[\int_{-\pi}^{\pi}\left|\frac{\Delta_{t}^{j} f(x)}{I(t)}\right|^{a} d x\right]^{p / a} \frac{d t}{t}\right\}^{1 / p} . \\
Y_{a, j}\left(t, c_{n}\right)=\left\{\sum_{n=-\infty}^{\infty}\left|c_{n}\right|^{a}|\sin n t / 2|^{a j}\right\}^{1 / a} \cdot \\
\tilde{A}_{a, j, I}\left(c_{n}\right)=\left\{\int_{0}^{1}\left|\frac{Y_{a, j}\left(t, c_{n}\right)}{I(t)}\right|^{p} \frac{d t}{t}\right\}^{1 / p} \cdot \\
\left\{\left(w_{n}\right): w_{n}>0, w_{n}=w_{-n}, w_{|n|} \downarrow,\left\|w_{n}\right\|_{1}=\sum_{n=-\infty}^{\infty} w_{n}<\infty\right\}, \\
{ }_{a}\left\|c_{n}\right\|_{p, w}=\left\{\sum_{n=-\infty}^{\infty}\left|c_{n}\right|^{a}\left(w_{n}\right)^{1-a / p}\right\}^{1 / a}, \\
\left\|c_{n}\right\|_{p}=\inf _{\left(w_{n}\right) \in W}\left[\left\|w_{n}\right\|_{1}^{1 / p-1 / a}{ }_{a}\left\|c_{n}\right\|_{p, w}\right] . \\
a_{a} l_{p}=\left\{\left(c_{n}\right):{ }_{a}\left\|c_{n}\right\|_{p}<\infty\right\} .
\end{gathered}
$$

By Hölder's inequality, we see that $\left\|c_{n}\right\|_{p} \leq_{a}\left\|c_{n}\right\|_{p}$ if $0<p \leq a<\infty$. In particular, $\left\|c_{n}\right\|_{p}={ }_{p}\left\|c_{n}\right\|_{p}$. The above inequality holds for the case $0<p<\infty=a$. Because, we read

$$
{ }_{\infty}\left\|c_{n}\right\|_{p}=\inf _{\left(w_{n}\right)}\left[\left\|w_{n}\right\|^{1 / p} \sup _{n}\left(\left|c_{n}\right| w_{n}^{-1 / p}\right)\right]
$$

and we have

$$
\sum\left|c_{n}\right|^{p}=\sum\left(\left|c_{n}\right| w_{n}^{-1 / p}\right)^{p} w_{n} \leq\left\|w_{n}\right\|_{1}\left[\sup _{n}\left(\left|c_{n}\right| w_{n}^{-1 / p}\right)\right]^{p} .
$$

THEOREM 3. Let $1<a \leq 2,1 / a+1 / a^{\prime}=1,0<p \leq a^{\prime}$, and $j$ be $a$ positive integer. Then, we have

$$
a^{\prime}\left\|c_{n}|n|^{1 / a^{\prime}-1 / p}[I(1 /|n|)]^{-1}\right\|_{p} \leq K_{a} A_{p, j, I}(f) .
$$

The inequality holds also for the case $a=1$ and $0<p<\infty=a^{\prime}$. 
TheOREM 4. Let $1 \leq a \leq 2,0<p \leq a$, and $j$ be a positive integer. Suppose that a positive and increasing function $I(t)$ on $(0, \infty)$ satisfies the following conditions: There exist positive numbers $\varepsilon$ and $\delta$ such that

$$
\begin{gathered}
0<\varepsilon<1<\delta \\
\int_{0}^{t} u^{\beta}[I(u)]^{-a} d u \leq K t^{\beta+1}[I(t)]^{-a}
\end{gathered}
$$

where $\beta=-2+a / p+a j+\delta(1-a / p)$, and

$$
\int_{t}^{1} u^{\gamma}[I(u)]^{-a} d u \leq K t^{\gamma+1}[I(t)]^{-a}
$$

where $\gamma=-2+a / p+\varepsilon(1-a / p)$. Then, we have

$$
{ }_{a^{\prime}} A_{p, j, I}(f) \leq K_{a}\left\|c_{n}|n|^{1 / a-1 / p}[I(1 /|n|)]^{-1}\right\|_{p} \text {. }
$$

Proof of theorems shall be based on the following lemmata:

Lemma 2. Let $0<p \leq a<\infty$. Then, we have

$$
{ }_{a}\left\|c_{n}|n|^{1 / a-1 / p}[I(1 /|n|)]^{-1}\right\|_{p} \leq K_{a} \tilde{A}_{p, J, I}\left(c_{n}\right) .
$$

(In the norm of the left hand side, we omit the term for $n=0$.) The inequality holds for the case $0<p<\infty=a$.

Lemma 3. Let $0<p \leq a<\infty$. Suppose that a function I( $t$ ) satisfies (9) and (10). Then, we have

$$
{ }_{a} \tilde{A}_{p, j, I}\left(c_{n}\right) \leq K_{a}\left\|c_{n}|n|^{1 / a-1 / p}[I(1 /|n|)]^{-1}\right\|_{p} .
$$

Proof of Lemma 2. Let us discuss the case $0<p<a<\infty$ :

$$
\begin{aligned}
{\left[{ }_{a} \tilde{A}_{p, j, I}\left(c_{k}\right)\right]^{p} } & =\int_{0}^{1} t^{-1}[I(t)]^{-p}\left[Y_{a, j}\left(t, c_{k}\right)\right]^{p-a}\left[Y_{a, j}\left(t, c_{k}\right)\right]^{a} d t \\
& =\sum_{n=-\infty}^{\infty}\left|c_{n}\right|^{a} \int_{0}^{1} t^{-1}[I(t)]^{-p}\left[Y_{a, j}\left(t, c_{k}\right)\right]^{p-a}|\sin n t / 2|^{a j} d t \\
& =\sum_{n=-\infty}^{\infty}\left|c_{n}\right|^{a} M_{n}, \quad \text { say. }
\end{aligned}
$$

We put

$$
w_{n}=\int_{0}^{1 /|n|}[I(t)]^{-p}\left[Y_{a, j}\left(t, c_{k}\right)\right]^{p} d t
$$


Then, we have

$$
\begin{aligned}
\sum_{n=1}^{\infty} w_{n} & =\sum_{n=1}^{\infty} \sum_{m=n}^{\infty} \int_{1 /(m+1)}^{1 / m}[I(t)]^{-p}\left[Y_{a, j}\left(t, c_{k}\right)\right]^{p} d t \\
& =\sum_{m=1}^{\infty} \sum_{n=1}^{m} \int_{1 /(m+1)}^{1 / m}[I(t)]^{-p}\left[Y_{a, j}\left(t, c_{k}\right)\right]^{p} d t \\
& =\sum_{m=1}^{\infty} m \int_{1 /(m+1)}^{1 / m}[I(t)]^{-p}\left[Y_{a, j}\left(t, c_{k}\right)\right]^{p} d t \\
& \leq K \int_{0}^{1} t^{-1}[I(t)]^{-p}\left[Y_{a, j}\left(t, c_{k}\right)\right]^{p} d t \\
& \left.=K{ }_{a} \tilde{A}_{p, J, I}\left(c_{n}\right)\right]^{p}
\end{aligned}
$$

That is, we have

$$
\left(w_{n}\right) \in W \quad \text { and } \quad\left\|w_{n}\right\|_{1} \leq K\left[{ }_{a} \tilde{A}_{p, J, I}\left(c_{n}\right)\right]^{p}
$$

Let us put $P=a / p>1$ and $1 / P+1 / Q=1$. Then, by Hölder's inequality,

$$
\begin{aligned}
{\left[w_{n}\right]^{1 / Q}\left[M_{n}\right]^{1 / P} \geq } & \int_{0}^{1 /|n|}\left\{t^{-1}[I(t)]^{-p}\left[Y_{a, j}\left(t, c_{k}\right)\right]^{p-a}|\sin n t / 2|^{a J}\right\}^{1 / P} \\
& \times\left\{[I(t)]^{-p}\left[Y_{a, j}\left(t, c_{k}\right)\right]^{p}\right\}^{1 / Q} d t \\
= & \int_{0}^{1 /|n|} t^{-p / a}[I(t)]^{-p}|\sin n t / 2|^{p j} d t \\
\geq & |n|^{p / a-1}[I(1 /|n|)]^{-p} \int_{0}^{1} u^{-p / a}|\sin u / 2|^{p_{J}} d u
\end{aligned}
$$

where the last integral is a positive constant. So we have, for a positive constant $K$,

$$
M_{n} \geq K\left[w_{n}\right]^{1-a / p}|n|^{1-a / p}[I(1 /|n|)]^{-a} .
$$

Now we have

$$
\begin{aligned}
{\left[{ }_{a} \tilde{A}_{p, J, I}\left(c_{k}\right)\right]^{p} } & \geq K \sum_{n=-\infty}^{\infty}\left|c_{n}\right|^{a}\left\{|n|^{1 / a-1 / p}[I(1 /|n|)]^{-1}\right\}^{a}\left[w_{n}\right]^{1-a / p} \\
& =K_{a}\left\|c_{n}|n|^{1 / a-1 / p}[I(1 /|n|)]^{-1}\right\|_{p, w}^{a} .
\end{aligned}
$$


Therefore, we have

$$
\begin{aligned}
& \left\|w_{n}\right\|_{1}^{1 / p-1 / a}{ }_{a}\left\|c_{n}|n|^{1 / a-1 / p}[I(1 /|n|)]^{-1}\right\|_{p, w} \\
& \quad \leq K\left[{ }_{a} \tilde{A}_{p, j, I}\left(c_{k}\right)\right]^{p(1 / p-1 / a)}\left[{ }_{a} \tilde{A}_{p, j, I}\left(c_{k}\right)\right]^{p / a} \\
& \quad=K_{a} \tilde{A}_{p, j, I}\left(c_{k}\right),
\end{aligned}
$$

by which we have the conclusion for the case $0<p<a<\infty$.

For the case $0<p=a<\infty$, the result of Lemma 2 is easily seen. In fact, we have to show that

$$
\left\|c_{n} I(1 /|n|)^{-1}\right\|_{p}^{p} \leq\left[K_{p} \tilde{A}_{p, j, I}\left(c_{n}\right)\right]^{p} .
$$

However, the right hand side of the above inequality is

$$
\begin{aligned}
& \sum\left|c_{n}\right|^{p} \int_{0}^{1} t^{-1}[I(t)]^{-p}|\sin n t / 2|^{p j} d t \\
& \quad=\sum\left|c_{n}\right|^{p} \int_{0}^{|n|} u^{-1}[I(u /|n|)]^{-p}|\sin u / 2|^{p j} d u \\
& \quad \geq \sum\left|c_{n}\right|^{p} \int_{0}^{1} u^{-1}[I(u /|n|)]^{-p}|\sin u / 2|^{p j} d u \\
& \quad \geq \sum\left|c_{n}\right|^{p}[I(1 /|n|)]^{-p} \int_{0}^{1} u^{-1}|\sin u / 2|^{p j} d u,
\end{aligned}
$$

which is the required result.

Let us show the result for the case $0<p<\infty=a$. Set

$$
Y_{\infty, j}\left(t, c_{k}\right)=\sup _{k}\left(\left|c_{k}\right| \mid \sin k t / 2 \psi^{j}\right),
$$

and

$$
w_{n}=\int_{0}^{1 /|n|}[I(t)]^{-p}\left[Y_{\infty, j}\left(t, c_{k}\right)\right]^{p} d t
$$

then we have

$$
\left\|w_{n}\right\|_{1} \leq K\left[_{\infty} \tilde{A}_{p, j, I}\left(c_{n}\right)\right]^{p}
$$

We have to show that

$$
\left\|w_{n}\right\|_{1}^{1 / p}\left\|c_{n}|n|^{-1 / p}[I(1 /|n|)]^{-1}\right\|_{p, w} \leq K_{\infty} \tilde{A}_{p, j, I}\left(c_{n}\right) .
$$

Due to the inequality (11), the above inequality is equivalent to

$$
{ }_{\infty}\left\|c_{n}|n|^{-1 / p}[I(1 /|n|)]^{-1}\right\|_{p, w} \leq K
$$


which must be read as

$$
\left\|c_{n}|n|^{-1 / p}[I(1 /|n|)]^{-1} w_{n}^{-1 / p}\right\|_{\infty} \leq K
$$

Therefore we have to show that

$$
\begin{aligned}
& \left|c_{n}\right||n|^{-1 / p}[I(1 /|n|)]^{-1} \\
& \quad \leq K w_{n}^{1 / p}=K\left\{\int_{0}^{1 /|n|}[I(t)]^{-p} \sup _{k}\left(\left|c_{k}\right| \mid \sin k t / 2 \psi^{j}\right)^{p} d t\right\}^{1 / p},
\end{aligned}
$$

that is,

$$
|n|^{-1 / p}[I(1 /|n|)]^{-1} \leq K\left\{\int_{0}^{1 /|n|}[I(t)]^{-p}|\sin n t / 2|^{p j} d t\right\}^{1 / p} .
$$

However, the right hand side integral is

$$
\begin{aligned}
(1 /|n|) \int_{0}^{1}[ & I(u /|n|)]^{-p}|\sin u / 2|^{p j} d u \\
& \geq(1 /|n|)[I(1 /|n|)]^{-p} \int_{0}^{1}|\sin u / 2|^{p j} d u,
\end{aligned}
$$

which implies the required.

Proof of Lemma 3. Let us discuss firstly the case $0<p<a<\infty$.

For any sequence $\left(w_{n}\right) \in W$, there is a positive, decreasing, continuous and integrable function $w(t)$ on $(0, \infty)$ such that $w(n)=w_{n}$. For the numbers $\varepsilon$ and $\delta(0<\varepsilon<1<\delta)$, we may find a function $w^{*}(t)$ which satisfies the following properties:

(i) $w(t) \leq w^{*}(t)$;

(ii) $t^{\delta} w^{*}(t)$ is increasing;

(iii) $t^{\varepsilon} w^{*}(t)$ is decreasing; and

(iv) $\int_{0}^{\infty} w^{*}(t) d t=K \int_{0}^{\infty} w(t) d t$. (cf. A. Beurling [1].)

Let $P=a / p, 1 / P+1 / Q=1$. Then, by the Hölder inequality, we have

$$
\begin{aligned}
& {\left[{ }_{a} \tilde{A}_{p, j, I}\left(c_{n}\right)\right]^{p} } \\
&=\int_{0}^{1}\left\{\left[Y_{a, j}\left(t, c_{n}\right)\right]^{p}[I(t)]^{-p} t^{(-1+2 / Q)}\left[w^{*}(1 / t)\right]^{-1+p / a}\right\} \\
& \times\left\{t^{-2 / Q}\left[w^{*}(1 / t)\right]^{1-p / a}\right\} d t \\
& \leq\left\{\int_{0}^{1}\left[Y_{a, j}\left(t, c_{n}\right)\right]^{p P}[I(t)]^{-p P} t^{(-1+2 / Q) P}\left[w^{*}(1 / t)\right]^{(-1+p / a) P} d t\right\}^{1 / P} \\
& \times\left\{\int_{0}^{1} t^{-2}\left[w^{*}(1 / t)\right]^{Q(1-p / a)} d t\right\}^{1 / Q},
\end{aligned}
$$

where the last integral is less than $\left\|w^{*}\right\|_{1}=K\|w\|_{1}$. 
Hence we have

$$
\begin{aligned}
& {\left[{ }_{a} \tilde{A}_{p, j, I}\left(c_{n}\right)\right]^{p P}} \\
& \leq\left\|w^{*}\right\|_{1}^{P / Q} \int_{0}^{1}[I(t)]^{-a} t^{(-1+2 / Q) P}\left[w^{*}(1 / t)\right]^{1-a / p} \\
& \quad \times \sum_{n=-\infty}^{\infty}\left|c_{n}\right|^{a}|\sin n t / 2|^{a j} d t \\
& =\left\|w^{*}\right\|_{1}^{P / Q} \sum_{n=-\infty}^{\infty}\left|c_{n}\right|^{a} \int_{0}^{1}[I(t)]^{-a} t^{(-2+a / p)} \\
& \quad \times\left[w^{*}(1 / t)\right]^{1-a / p}|\sin n t / 2|^{a j} d t
\end{aligned}
$$

where the last integral will be denoted by $J$. We split the integral $J$ into two parts;

$$
J=\int_{0}^{1 /|n|}+\int_{1 /|n|}^{1}=J_{1}+J_{2}, \text { say. }
$$

Then

$$
\begin{aligned}
J_{1} \leq K|n|^{a j} \int_{0}^{1 /|n|}[I(t)]^{-a} t^{(-2+a / p)+a j}\left[w^{*}(1 / t)\right]^{1-a / p} d t \\
\leq K|n|^{a j} \int_{0}^{1 /|n|}[I(t)]^{-a} t^{(-2+a / p)+a j+\delta(1-a / p)} \\
\times \frac{1}{\left[\left(1 / t^{\delta}\right) w^{*}(1 / t)\right]^{-1+a / p}} d t .
\end{aligned}
$$

Since $\left(1 / t^{\delta}\right) w^{*}(1 / t)$ is decreasing and is greater than $|n|^{\delta} w^{*}(|n|)$, we have

$$
J_{1} \leq K|n|^{a j}\left[|n|^{\delta} w^{*}(|n|)\right]^{1-a / p} \int_{0}^{1 /|n|} t^{\beta}[I(t)]^{-a} d t
$$

where $\beta=-2+a / p+a j+\delta(1-a / p)$. According to the assumption, the above integral is estimated by $K|n|^{-\beta-1}[I(1 /|n|)]^{-a}$. Therefore, we have

$$
J_{1} \leq K|n|^{1-a / p}[I(1 /|n|)]^{-a}\left[w^{*}(|n|)\right]^{1-a / p}
$$


Now, we should estimate the second part $J_{2}$ :

$$
\begin{aligned}
J_{2} \leq K \int_{1 /|n|}^{1}[I(t)]^{-a} t^{(-2+a / p)}\left[w^{*}(1 / t)\right]^{1-a / p} d t \\
=K \int_{1 /|n|}^{1}[I(t)]^{-a} t^{(-2+a / p)-\varepsilon(-1+a / p)} \\
\times \frac{1}{\left[\left(1 / t^{\varepsilon}\right) w^{*}(1 / t)\right]^{-1+a / p}} d t .
\end{aligned}
$$

Since $\left(1 / t^{\varepsilon}\right) w^{*}(1 / t)$ is increasing and greater than $|n|^{\varepsilon} w^{*}(|n|)$, we have

$$
J_{2} \leq K\left[|n|^{\varepsilon} w^{*}(|n|)\right]^{1-a / p} \int_{1 /|n|}^{1} t^{\gamma}[I(t)]^{-a} d t,
$$

where $\gamma=-2+a / p+\varepsilon(1-a / p)$. According to the assumption, the last integral is estimated by $K|n|^{-\gamma-1}[I(1 /|n|)]^{-a}$. Therefore we have the same estimate for $J_{2}$ as for $J_{1}$. Using the above estimation for $J$, we have

$$
\begin{aligned}
& {\left[{ }_{a} \tilde{A}_{p, j, I}\left(c_{n}\right)\right]^{p P} \leq K\left\|w_{n}^{*}\right\|_{1}^{P / Q} \sum_{n=-\infty}^{\infty}\left|c_{n}\right|^{a} J} \\
& \quad \leq K\left\|w_{n}^{*}\right\|_{1}^{P / Q} \sum_{n=-\infty}^{\infty}\left|c_{n}\right|^{a}|n|^{1-a / p}[I(1 /|n|)]^{-a}\left[w^{*}(|n|)\right]^{1-a / p} .
\end{aligned}
$$

Since $\left\|w^{*}\right\|_{1}=K\|w\|_{1}$ and $w(|n|) \leq w^{*}(|n|)$, we have

$$
\begin{aligned}
{ }_{a} \tilde{A}_{p, j, I}\left(c_{n}\right) \leq & K\left\|w_{n}\right\|_{1}^{1 / p-1 / a} \\
& \times\left\{\sum_{n=-\infty}^{\infty}\left|c_{n}\right|^{a}|n|^{a(1 / a-1 / p)}[I(1 /|n|)]^{-a} w(|n|)^{1-a / p}\right\}^{1 / a} \\
= & K\left\|w_{n}\right\|_{1}^{1 / p-1 / a}{ }_{a}\left\|c_{n}|n|^{(1 / a-1 / p)}[I(1 /|n|)]^{-1}\right\|_{p, w} .
\end{aligned}
$$

Therefore, we have Lemma 3 for $0<p<a<\infty$.

For the case $p=a$, we have to show that

$$
\left[{ }_{p} \tilde{A}_{p, j, I}\left(c_{n}\right)\right]^{p} \leq K\left\|c_{n}[I(1 /|n|)]^{-1}\right\|_{p}^{p} .
$$

In fact, the left hand side of the above is

$$
\sum\left|c_{n}\right|^{p} \int_{0}^{1} t^{-1}[I(t)]^{-p}|\sin n t / 2|^{p j} d t
$$

where the integral part is less than

$$
\int_{0}^{1 /|n|} t^{-1}[I(t)]^{-p}|n t|^{p j} d t+\int_{1 /|n|}^{1} t^{-1}[I(t)]^{-p} d t \leq K[I(1 /|n|)]^{-p},
$$


by which we have the conclusion. (In the above calculation, we have used the inequalities (9) and (10) with $a=p$.)

Proof of Theorem 3. The Fourier series of $\Delta_{t}^{j} f(x)$ is

$$
\Delta_{t}^{J} f(x) \sim K \sum_{n=-\infty}^{\infty} c_{n} e^{i n x}\left(e^{i n t}-1\right)^{j}
$$

Therefore, by the Hausdorff-Young inequality, we have

$$
Y_{a^{\prime}, J}\left(t, c_{n}\right) \leq K\left\|\Delta_{t}^{j} f(\cdot)\right\|_{a},
$$

and hence,

$$
{ }_{a} \tilde{A}_{p, J, I}\left(c_{n}\right) \leq K_{a} A_{p, j, I}(f) .
$$

Then, apply Lemma 2, we have the conclusion.

Proof of Theorem 4. By the Hausdorff-Young inequality, we have

$$
\left\|\Delta_{t}^{j} f(\cdot)\right\|_{a^{\prime}} \leq K Y_{a, j}\left(t, c_{n}\right) .
$$

(The above inequality also holds for the case $a=1, a^{\prime}=\infty$.) Therefore, we have

$$
{ }_{a^{\prime}} A_{p, j, I}(f) \leq K_{a} \tilde{A}_{p, J, I}\left(c_{n}\right) .
$$

By Lemma 3, the proof is complete.

From Theorems 3 and 4, we have

Theorem 5. Let $0<p \leq 2$. Suppose $I(t)$ satisfies (9) and (10) for $a=2$. Then, ${ }_{2} A_{p, J, I}(f)<\infty$ if and only if

$$
{ }_{2}\left\|c_{n}|n|^{1 / 2-1 / p}[I(1 /|n|)]^{-1}\right\|_{p}<\infty .
$$

4. We shall give several remarks:

In the definition of the shrivel norm ${ }_{a} \mathbf{A}_{p, J, \alpha}(f)$ in $\S 1$, let us replace the factor $t^{\alpha}$ by $I(t)$; then we have a general shrivel norm ${ }_{a} \mathbf{A}_{p, J, I}(f)$. (In the case, the difference $\Delta_{t}^{J} f(x)$ should be read as the symmetric difference.) Through the similar discussion as in the previous section, we can show that ${ }_{2} \mathbf{A}_{p, J, I}(f)<\infty$ if and only if ${ }_{2} A_{p, J, I}(f)<\infty$, under the same assumption of Theorem 5 .

Some discussion on general B-type and C-type norms (cf. §2) may be found in the paper by R. G. Mamedov and G. I. Osmanov [7], and by M. and $\mathrm{S}$. Izumi [3]. 
We have shown that the norms ${ }_{2} A_{p, j, I}(f)$ are, up to equivalence, independent of the choice of the difference order $j$, indirectly through the result of the $\S 3$. However, this can be shown by the argument due to $\mathrm{C}$. Herz ([2]; Appendix 1.)

C. J. Neugenbauer's result (Theorem 2 in [8]) is quite similar to our Theorem 3.

Similar discussions can be used for the case of Fourier transforms in the $n$-dimensional Euclidean space. Cf. Kinukawa [6].

\section{REFERENCES}

[1] A. Beurling, Construction and analysis of some convolution algebras, Ann. Inst. Fourier, 14 (1964), 1-32.

[2] C. S. Herz, Lipschitz spaces and Bernstein's theorem on absolutely convergent Fourier transforms, J. Math. and Mech., 18 (1968), 283-323.

[3] M. and S. Izumi, Lipschitz classes and Fourier coefficients, J. Math. and Mech., 18 (1969), 857-870.

[4] M. Kinukawa, Lipschitz spaces and Fourier series, (in Japanese), Sugaku (published by Math. Soc. of Japan), 24 (1972), 51-53.

[5] Contractions of Fourier transforms in $R_{k}$, Tohoku Math. J., 24 (1972), 233-243.

[6] Generalized Lipschitz class of functions and their Fourier transforms, to appear.

[7] R. G. Mamedov and G. I. Osmanov, Some properties of functions expressible by their Fourier coefficients and transforms, Izvestiya VUZ. Matematika, 20 (1976), 65-78.

[8] C. J. Neugebauer, The $L^{p}$ modulus of continuity and Fourier series of Lipschitz functions, Proc. Amer. Math. Soc., 64 (1977), 71-76.

[9] G. Sunouchi, On convolution algebra of Beurling, Tohoku Math. J., 19 (1967), 303-310.

[10] B. S. Yadav, Contractions of functions and their Fourier series, Pacific J. Math., 31 (1969), 827-833.

Received March 26, 1982.

INTERNATIONAL CHRISTIAN UNIVERSITY

MitaKa, TOKYO, JAPAN 181 


\section{PACIFIC JOURNAL OF MATHEMATICS}

EDITORS

Donald BABBITt (Managing Editor)

University of California

Los Angeles, CA 90024

Hugo Rossi

University of Utah

Salt Lake City, UT 84112

C. C. Moore and Arthur Ogus

University of California

Berkeley, CA 94720

\section{J. DugundiI}

Department of Mathematics

University of Southern California

Los Angeles, CA 90089-1113

R. Finn and H. SAMELSON

Stanford University

Stanford, CA 94305

\section{ASSOCIATE EDITORS}
R. ARENS
E. F. BECKENBACH
B. H. NEUMANN
F. WOLF
K. YOSHIDA (1906-1982)

\section{SUPPORTING INSTITUTIONS}

UNIVERSITY OF ARIZONA

UNIVERSITY OF BRITISH COLUMBIA

CALIFORNIA INSTITUTE OF TECHNOLOGY

UNIVERSITY OF CALIFORNIA

MONTANA STATE UNIVERSITY

UNIVERSITY OF NEVADA. RENO

NEW MEXICO STATE UNIVERSITY

OREGON STATE UNIVERSITY
UNIVERSITY OF OREGION

UNIVERSITY OF SOUTHERN CALIFORNIA

STANFORD UNIVERSITY

UNIVERSITY OF HAWAII

UNIVERSITY OF TOKYO

UNIVERSITY OF UTAH

WASHINGTON STATE UNIVERSITY

UNIVERSITY OF WASHINGTON 


\section{Pacific Journal of Mathematics}

Vol. 109, No. $1 \quad$ May, 1983

Donald George Babbitt and V. S. Varadarajan, Formal reduction theory of meromorphic differential equations: a group theoretic view $\ldots \ldots \ldots \ldots 1$

Jo-Ann Deborah Cohen, Norms on $F(X) \ldots \ldots \ldots \ldots \ldots \ldots \ldots \ldots \ldots \ldots$

Robert Fitzgerald, Witt kernels of function field extensions $\ldots \ldots \ldots \ldots \ldots 89$

Hervé Jacquet and Joseph Andrew Shalika, The Whittaker models of induced representations .............................. 107

Masakiti Kinukawa, Some generalizations of contraction theorems for

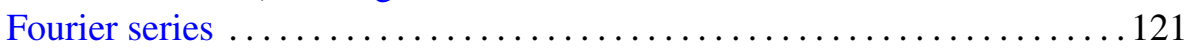

Joseph Weston Kitchen, Jr. and David A. Robbins, Sectional

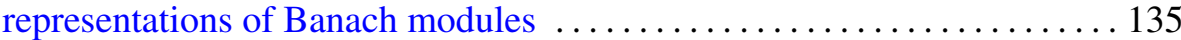

Victor Charles Pestien, Jr., Weak approximation of strategies in measurable gambling

Richard Scott Pierce and Charles Irvin Vinsonhaler, Realizing central

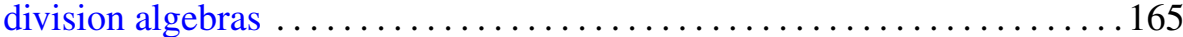

Walter Ricardo Ferrer Santos, Cohomology of comodules ............ 179

Marko Tadić, Harmonic analysis of spherical functions on reductive groups

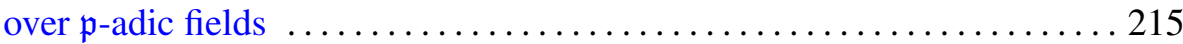

Lorenzo Traldi, The determinantal ideals of link modules. II . . . . . . . . 237

Alain J. Valette, A remark on the Kasparov groups $\operatorname{Ext}^{i}(A, B) \ldots \ldots \ldots 247$ 